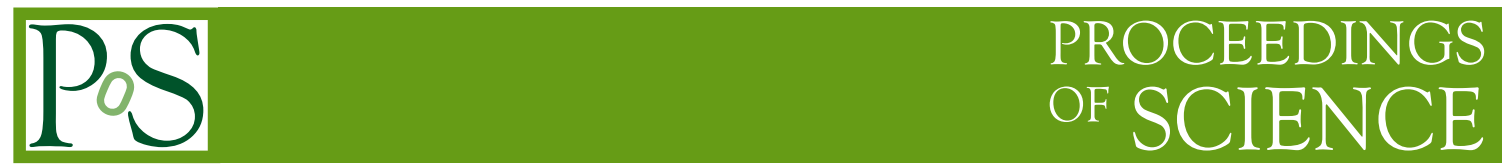

\title{
Review of BSM neutral Higgs results in CMS
}

\author{
Alberto Zucchetta*, on behalf of the CMS collaboration \\ INFN and Università di Padova \\ E-mail: a.zucchetta@cern.ch
}

Searches for beyond the standard model neutral Higgs bosons at the CMS experiment are presented. The analyses are based on proton-proton collision data recorded by the CMS experiment at $\sqrt{s}=7 \mathrm{TeV}$ and $\sqrt{s}=8 \mathrm{TeV}$ center-of-mass energy in 2011 and 2012, corresponding to integrated luminosities of up to $4.9 \mathrm{fb}^{-1}$ and $19.7 \mathrm{fb}^{-1}$. No evidence for additional Higgs bosons other than the $125 \mathrm{GeV}$ standard model-like Higgs boson are found, and stringent exclusion limits are derived.

Prospects for Charged Higgs Discovery at Colliders - CHARGED 2014,

16-18 September 2014

Uppsala University, Sweden

${ }^{*}$ Speaker. 


\section{Introduction}

A Higgs boson of mass $125 \mathrm{GeV}$ has been discovered at the LHC [1, 2], with cross sections, branching fractions and CP quantum numbers compatible with the standard model (SM) predictions. Several extensions of the SM, such as supersymmetric theories, predict the presence of additional bosons. In the Two Higgs Doublet Model (2HDM) [4], a second Higgs doublet is introduced, giving rise to five physical Higgs boson states, one pseudoscalar $(A)$, two scalars $(h, H)$, and two charged $\left(\mathrm{H}^{+}, \mathrm{H}^{-}\right)$. Different types of 2HDM are present, depending on the couplings of leptons and quarks to the two doublets. The Minimal Supersymmetric Standard Model (MSSM) [5] is a particular type of $2 \mathrm{HDM}$ and the minimal extension of the SM involving supersymmetry. Only two free parameters can determine the masses of the Higgs bosons at tree level, usually $\tan \beta$, the ratio of the vacuum expectation values of the two doublets, and the mass of the pseudoscalar boson $m_{A}$. The mass of the lightest scalar Higgs boson $h$ is bound to be below the $Z$ mass, and in order to achieve $m_{h}=125 \mathrm{GeV}$, sizable radiative corrections are needed, requiring a large stop mass and/or mixing. The need for fine-tuning is solved in the Next-to-Minimal Supersymmetric Standard Model (NMSSM) [7]. The presence of an additional complex singlet field allows to achieve a mass of $125 \mathrm{GeV}$ for the lightest scalar Higgs boson, by means of mixing between the Higgs doublet and singlet fields. An additional scalar and one pseudoscalar bosons are predicted by NMSSM. Other theories $[8,9,10]$ predict new resonances decaying with final states involving the presence of the SM Higgs boson in the decay chain, or Higgs boson decays forbidden in the SM [11]. Searches for neutral Beyond Standard Model (BSM) Higgs bosons are presented, based on proton-proton collision data recorded by the CMS experiment [3] at $\sqrt{s}=7 \mathrm{TeV}$ and $\sqrt{s}=8 \mathrm{TeV}$ in 2011 and 2012 , respectively, corresponding to integrated luminosities of up to $4.9 \mathrm{fb}^{-1}$ and $19.7 \mathrm{fb}^{-1}$.

\section{MSSM searches in the $\tau \tau$ channel}

At the LHC, the neutral MSSM Higgs bosons, collectively denoted as $\phi$, are expected to be predominantly produced either in gluon-gluon fusion or in $b$-quark associated production. For larger values of $\tan \beta$ the latter mode dominates, and the branching fraction $B(\phi \rightarrow \tau \tau)$ is also enhanced. CMS has performed a search for $\phi \rightarrow \tau \tau$ using the full $25 \mathrm{fb}^{-1}$ of the 7 and $8 \mathrm{TeV}$ data and considering $\tau$ decays into electrons, muons and hadrons $\left(\tau_{h}\right)$ in five final states: $e \mu, e \tau_{h}, \mu \tau_{h}$, $\mu \mu$ and $\tau_{h} \tau_{h}$ [12]. The good $\tau$ reconstruction and identification performance, together with the relatively small SM backgrounds, makes this channel one of the most sensitive to signatures of an extended Higgs sector. Events are collected using a combination of $e, \mu$, and hadronic tau triggers, whose criteria varied during the data-taking periods. Offline, events are selected requiring two oppositely charged, isolated leptons, and split into two categories of either 0 or $\geq 1 b$-tagged jets to enhance the sensitivity to the two production modes. The invariant mass $m_{\tau \tau}$ is reconstructed from the leptons and the missing energy in the event using a likelihood technique based on a model of the $\tau$-decay phase-space and the missing energy resolution, which results into a typical $20 \%$ mass resolution. Model-independent upper limits at 95\% Confidence Level (CL) are derived on the resonance production rate $\sigma(g g \phi) B(\tau \tau)$ and $\sigma(b b \phi) B(\tau \tau)$ independently (Fig. 1), and interpreted as constraints on the MSSM parameters in the $m_{A}-\tan \beta$ space assuming the other MSSM parameters to be defined by the benchmark scenario [6]. In all cases, the signal hypothesis is tested against a background plus SM-Higgs hypothesis, taking into account the Higgs boson at $m_{h}=125 \mathrm{GeV}$. 

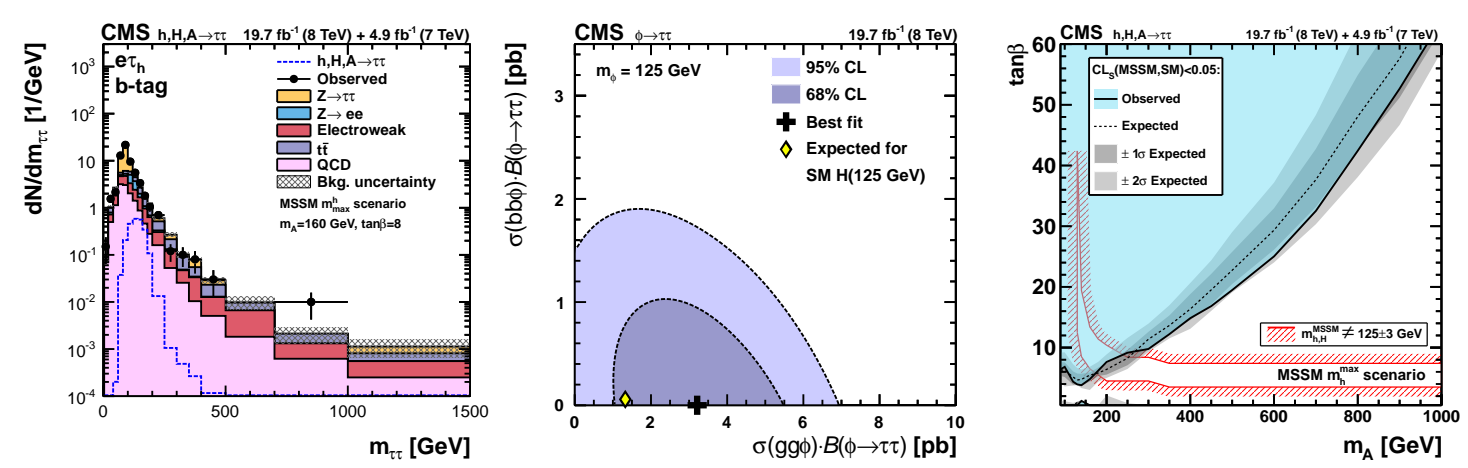

Figure 1: Left: observed and predicted $m_{\tau \tau}$ mass distributions in the $e \tau$ channel and $b$-tag category. Center: Likelihood contours of $\sigma(g g \phi) B(\tau \tau)$ versus $\sigma(b b \phi) B(\tau \tau)$, with the best fit value (cross) and the SM expectation for a $125 \mathrm{GeV}$ Higgs (diamond). Right: limit in the MSSM $m_{A}$-tan $\beta$ plane in the $m_{h}^{\max }$ scenario [12].

\section{MSSM searches in the $\mathbf{b} \bar{b}$ channel}

The search of low-mass MSSM Higgs bosons in the all-hadronic [13] channel is based on a data sample collected in 2011, making use of jet triggers with an online $b$-tagging algorithm, or the frequent presence of a soft muon in the event for the semi-leptonic [14] channel. Both analyses require at least three jets $b$-tagged in the offline selections, in order to exploit the presence of at least one physical $b$-quark in the associated production process. The all-hadronic analysis derives a background model from templates built from the different combinations of the three jets being tagged, where the signal yield is small. The signal fraction is extracted from a two-dimensional fit to the invariant mass distribution of the two leading jets and an additional variable that takes into account the number of tagged jets. The semi-leptonic analysis builds $b$-tagging probability matrices for the third jet fitting two discriminating variables, and reweights the double-tagged data spectrum to predict the three-tagged distribution. Another independent data-driven method, based on a customized nearest-neighbor algorithm, predicts the background shape in the three-tagged sample using events with one $b$-tag. The upper limits of the two channels are combined [15]; results are reported in Fig. 2. No significant deviation with respect to the SM background is observed, not confirming the excess of events seen by Tevatron for $m_{A}$ values around $150 \mathrm{GeV}$ [16].

\section{MSSM searches in the $\mu \mu$ channel}

The search of the $\phi \rightarrow \mu^{+} \mu^{-}$process [17] has been so far performed on the 2011 data sample. Candidates are experimentally characterized by the presence of two isolated, oppositely-charged muons, whose invariant mass would correspond to the mass of the boson. Events are assigned to three exclusive categories, two of them sensitive to $b$-quarks form the associated production, requiring one additional soft muon or a $b$-tagged jet, and the other enhancing the sensitivity to the gluon-gluon fusion production process. The search for a Higgs boson signal and the exclusion limit calculation is performed fitting the invariant mass spectrum in the three categories, and the limit on the rate is interpreted as a limit on the cross section times branching ratio, and projected on the MSSM $m_{A}$-tan $\beta$ parameter space in the $m_{h}$-max benchmark scenario (Fig. 2). 

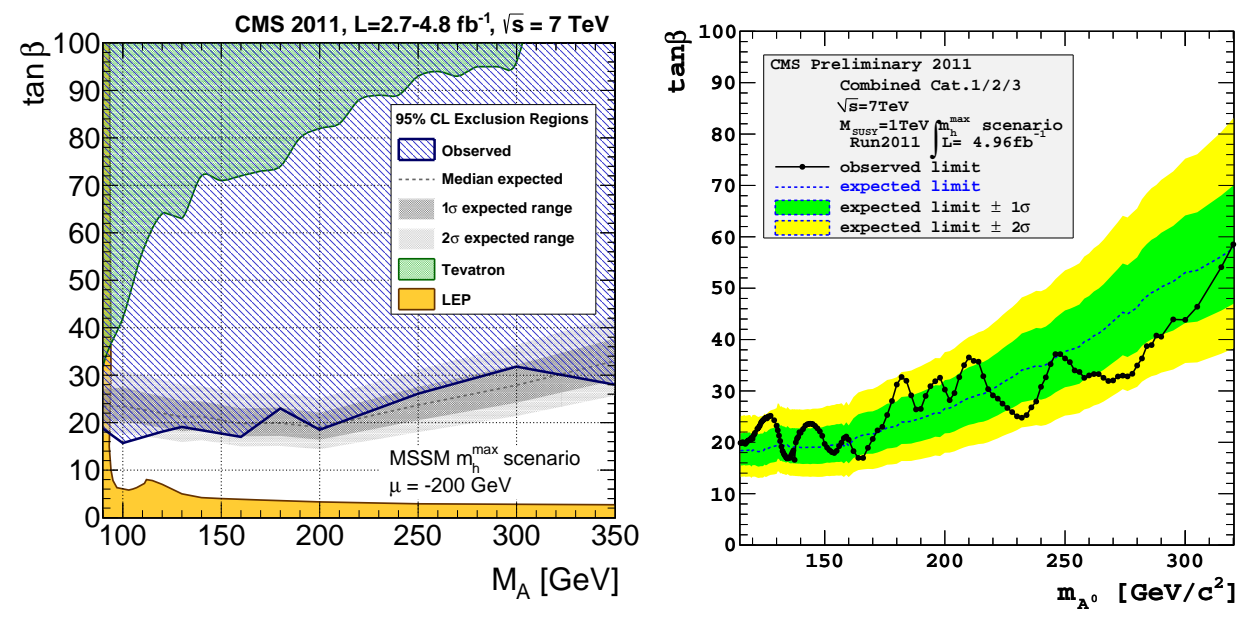

Figure 2: Left: Observed upper limits on $\tan \beta$ as a function of $M_{A}$, in the $m_{h}^{\max }$ benchmark scenario with the MSSM Higgsino parameter $\mu=-200 \mathrm{GeV}$ for the combined all-hadronic and semileptonic $b \phi \rightarrow 3 b$ results. Previous exclusion regions from LEP and Tevatron in the multi-b jet channel are overlaid [15]. Right: the combined exclusion limit for the $\phi \rightarrow \mu^{+} \mu^{-}$in the $m_{A}$-tan $\beta$ plane for the $m_{h}^{\max }$ benchmark scenario [17].

\section{NMSSM search for $h \rightarrow a_{1} a_{1} \rightarrow 4 \mu$ decays}

A search for light NMSSM Higgs bosons $a_{1}$ in the mass range $0.25<m_{a}<3.55 \mathrm{GeV}$ (below $2 \cdot m_{\tau}$ ) is performed on $20.7 \mathrm{fb}^{-1}$ of data collected at $\sqrt{s}=8 \mathrm{TeV}$ in the channel where a pair of $a_{1}$ bosons decays to four muons [18]. The analysis is not restricted to a specific model, and the results of the search are applicable to a broad spectrum of new physics scenarios. Events are selected by requiring the presence of two pairs of muons with $p_{T}>8 \mathrm{GeV}$ and at least one muon with $p_{T}>17 \mathrm{GeV}$ to fulfill the trigger requirements. Each pair is required to contain two isolated, opposite-charged muons with $m_{\mu \mu}<3.55 \mathrm{GeV}$. To enter in the signal region, the masses of both pairs have to be compatible within the experimental resolution. The background, consisting mainly in $b$-quarks decay into muons and $J / \Psi$ production, is estimated on data using sidebands events off the diagonal in Fig. 3. One event is observed in the signal region, while $3.8 \pm 1.2$ events are expected. Stringent limits at 95\% CL on $\sigma(p p \rightarrow 2 a) \times 2 \cdot \operatorname{Br}(a \rightarrow 2 \mu)$ are set.
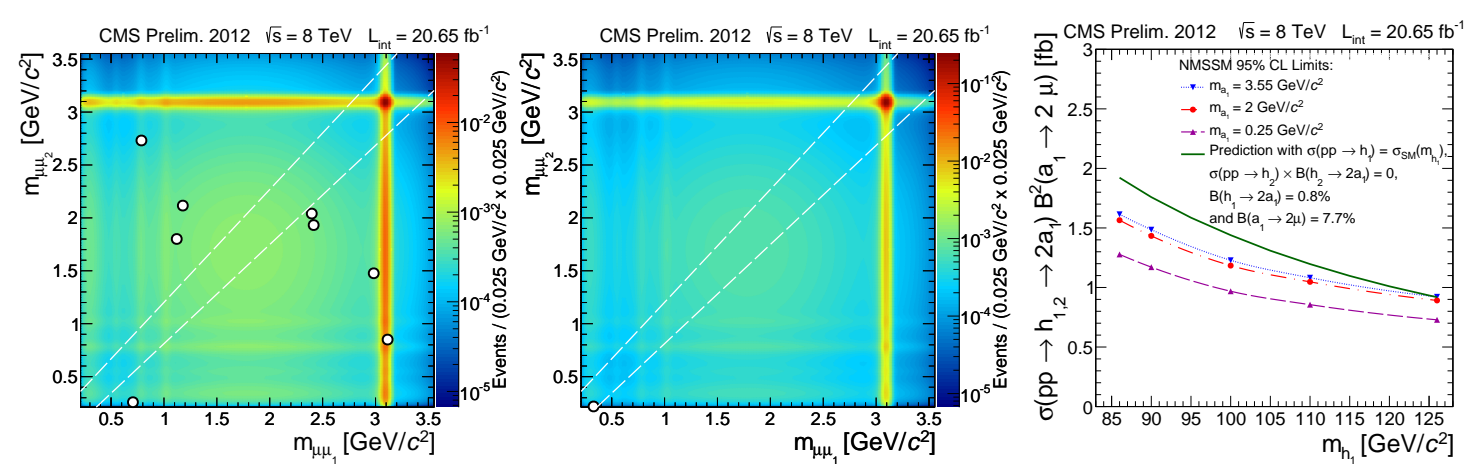

Figure 3: Expected and observed (white dots) in the 2D $m_{\mu \mu 1}$ vs $m_{\mu \mu 2}$ distribution in the sideband (left) and signal region (center). Right: Exclusion limit on the Higgs production cross section under specific cross section and branching ratio assumptions [18]. 


\section{HDM search for $\mathrm{H} \rightarrow \mathrm{hh}, \mathrm{A} \rightarrow \mathrm{Zh}$ with multileptons and photons}

The analysis targets final states with three or more leptons or a pair of resonant photons, to look for signatures of additional heavy Higgs bosons predicted by several BSM theories such as the 2HDM [19]. Leptons are produced by the $H \rightarrow h h$ and $A \rightarrow Z h$ decays, where one of both the light Higgs bosons decay into a pair of photons, or pairs of $W$ or $Z$ bosons, which in turn decay leptonically. Events are divided in several categories, depending on the number of $e, \mu$ and $\tau_{h}$, and if a $Z$ boson candidate can be reconstructed with a compatible invariant mass. Additional bins take into account the presence of $b$-tagged jets from $h \rightarrow b \bar{b}$, or large missing energy from $Z \rightarrow v v$ decays. The contamination from misidentified leptons and photon conversions is estimated on data, and SM backgrounds are taken from simulation. Events with at least a pair of photons and at least one additional isolated lepton are treated separately, and backgrounds are estimated using $m_{\gamma \gamma}$ sidebands. One category (Fig. 4 left) shows a small excess with a local (global) p-value of $1.5 \%(5 \%)$, but no significant deviations are found elsewhere. 95\% CL limits are extracted by simultaneously fitting all the categories, and model-independent limits are reported for the $H \rightarrow h h$ and $A \rightarrow Z h$, assuming SM-like branching fractions for the light Higgs boson. The exclusion is reported in the 2HDM parameter space as a function of $\cos (\beta-\alpha)$ (with $\alpha$ being the mixing angle of the two Higgs doublets) and $\tan \beta$ for a fixed $m_{A}$, and shown in Fig. 5.
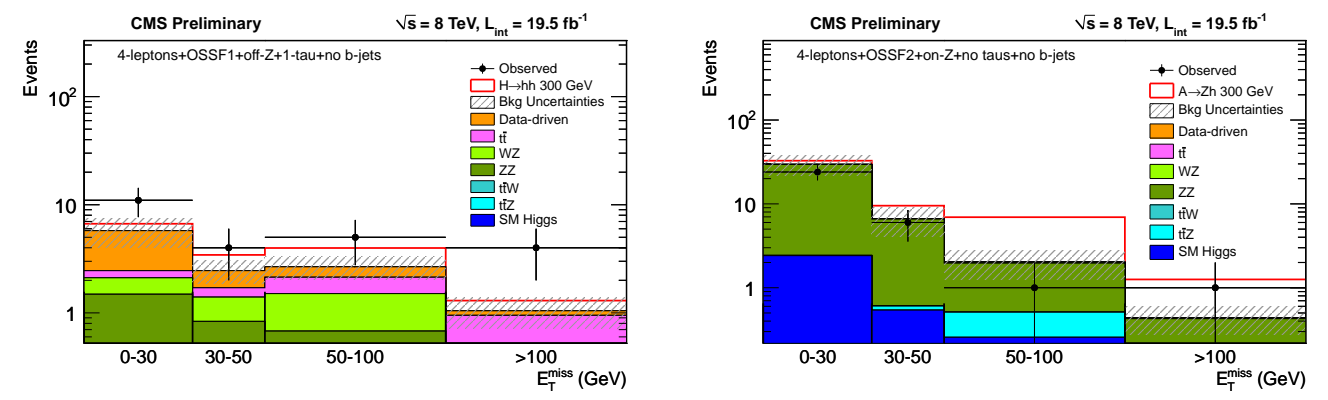

Figure 4: Signal and background expectation in the 4 lepton + OSSF1 off- $Z+1$ hadronic Tau + no $b$-jet channel (left), and in the 4 lepton + OSSF2 on- $Z+$ no hadronic Tau + no $b$-jet (right) [19].
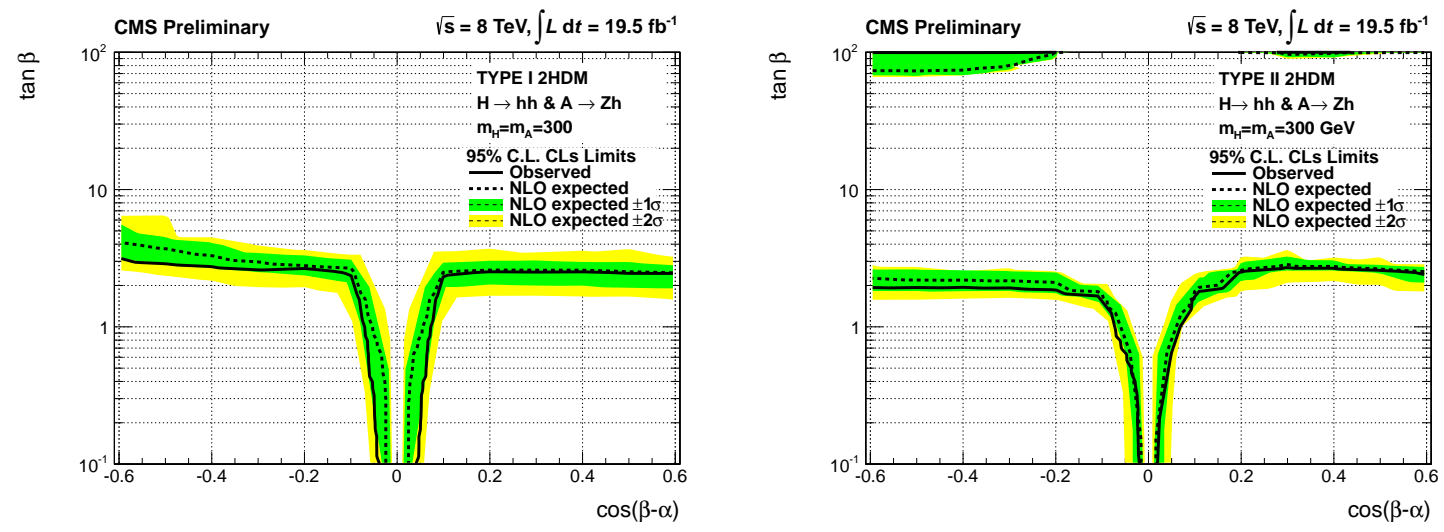

Figure 5: Observed and expected limits with 1- and 2- $\sigma$ bands in the $\cos (\beta-\alpha)-\tan \beta$ plane, for Type-I (left) and Type-II 2HDM (right) and $m_{A}=m_{H}=300 \mathrm{GeV}$ [19]. 


\section{Extra Dimension searches}

While the cross section of Higgs boson pair production is too small in the SM to be detected with the available data, several BSM theories predict narrow high-mass resonances decaying into pairs of SM Higgs bosons. One of them is the radion in Randall-Sundrum (RS1) models [8] of Warped Extra Dimensions (WED), which is the scalar field with a non-zero vacuum expectation value introduced to stabilize the radius of the extra dimension. A phenomenological relevant spin2 resonance could be the first excitation of the Kaluza-Klein (KK) graviton [9]. Two searches for resonances of Higgs boson pairs (denoted as $X$ ) have been performed with the full 2012 luminosity, targeting two different final states, $X \rightarrow h h \rightarrow \gamma \gamma b \bar{b}$ and $X \rightarrow h h \rightarrow b \bar{b} b \bar{b}$.

$\mathbf{X} \rightarrow \mathbf{h h} \rightarrow \gamma \gamma \mathbf{b} \overline{\mathbf{b}}$

The analysis dedicated to the search for the $X \rightarrow h h \rightarrow \gamma \gamma b \bar{b}$ process [20], a fully reconstructible final state, combines the large $h \rightarrow b \bar{b}$ branching fraction, with the low background, excellent mass resolution and ease of triggering due to the presence of two high- $p_{T}$ photons from the $h \rightarrow \gamma \gamma$ decay. Upon the two Higgs boson candidates, the four-body invariant mass $\left(m_{\gamma \gamma b \bar{b}}\right)$ is thereby calculated as the sum of the four product decays. A categorization is performed depending on the number of $b$-tagged jets. Two different strategies are adopted for the low mass $\left(m_{X} \lesssim 400\right.$ $\mathrm{GeV})$ and high mass scenarios $\left(m_{X}>400 \mathrm{GeV}\right)$. In the low mass, a cut-based selection is applied to $m_{j j}$ and $m_{\gamma \gamma b \bar{b}}$, and a fit is performed on the $m_{\gamma \gamma}$ spectrum. In the high mass scenario, after selections on $m_{\gamma \gamma}$ and $m_{j j}$, and a kinematic fit is applied to the two jets to improve the 4-body mass resolution (Fig. 6). The $m_{\gamma \gamma b \bar{b}}$ is then fitted looking for peaks above the background.
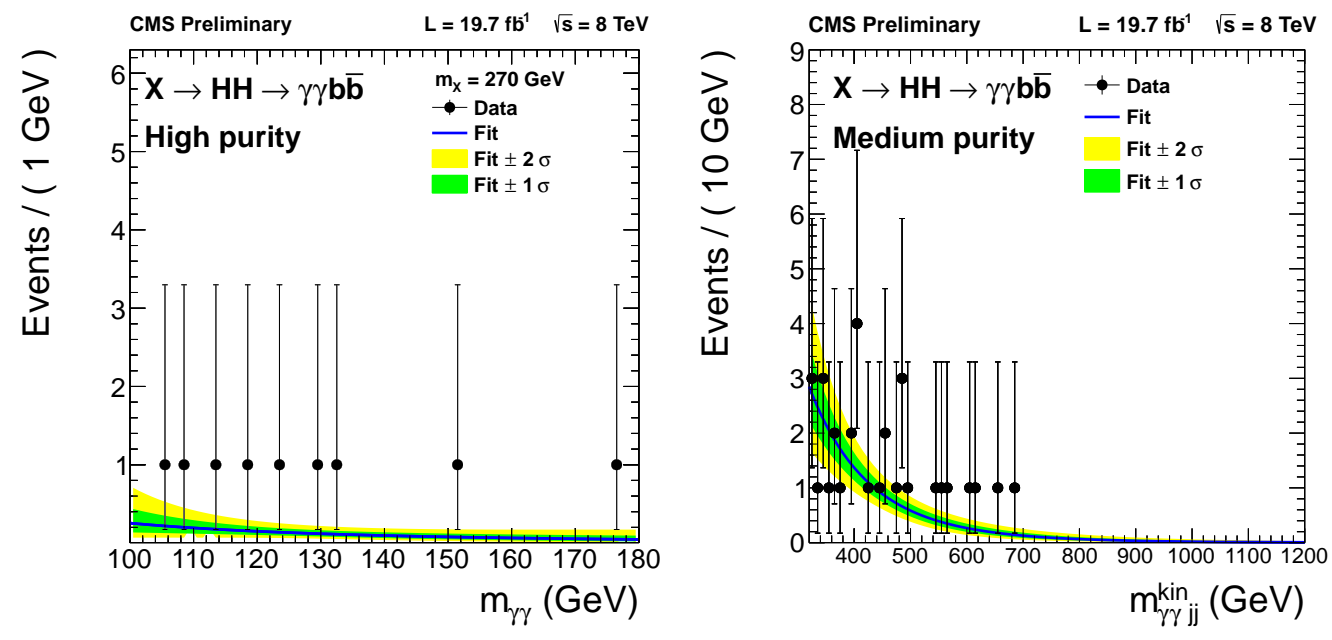

Figure 6: Left: $m_{\gamma \gamma}$ spectrum in the Low Mass region. Right: $m_{\gamma \gamma b b}$ in the High Mass region [20].

\section{$\mathbf{X} \rightarrow \mathbf{h h} \rightarrow \mathbf{b} \overline{\mathbf{b}} \mathbf{b} \overline{\mathbf{b}}$}

The final state of the $X \rightarrow h h \rightarrow b \bar{b} b \bar{b}$ analysis [21] is composed by four $b$-jets, making it difficult to be distinguished from the multijet background. Data are selected by using online $b$-tagging to maintain an acceptable rate. Once at least four jets in the event are selected and identified as 
originating from $b$-quarks, intermediate $h$ candidates are built with two different modes, depending on the mass region. In the low mass scenario, the jet pair with the closest invariant mass to the Higgs boson mass $\left(\left|m_{j j}-125 \mathrm{GeV}\right|<35 \mathrm{GeV}\right)$ are selected, while at high mass the two jets pair have to be close with each other $\left(\Delta R_{j j}<1.5\right)$. A Signal Region (SR) is defined as the circle with radius $17.5 \mathrm{GeV}$ around the $125 \mathrm{GeV}$ value in the plane of the two dijet pairs invariant mass (Fig. 7). Signal Sidebands (SB), together with Validation Region and Sidebands (VR, VB) are used to check the modeling of the multijet background. A kinematic constraint on the invariant mass of the Higgs boson candidates to $125 \mathrm{GeV}$ is used to improve the heavy resonance mass resolution.
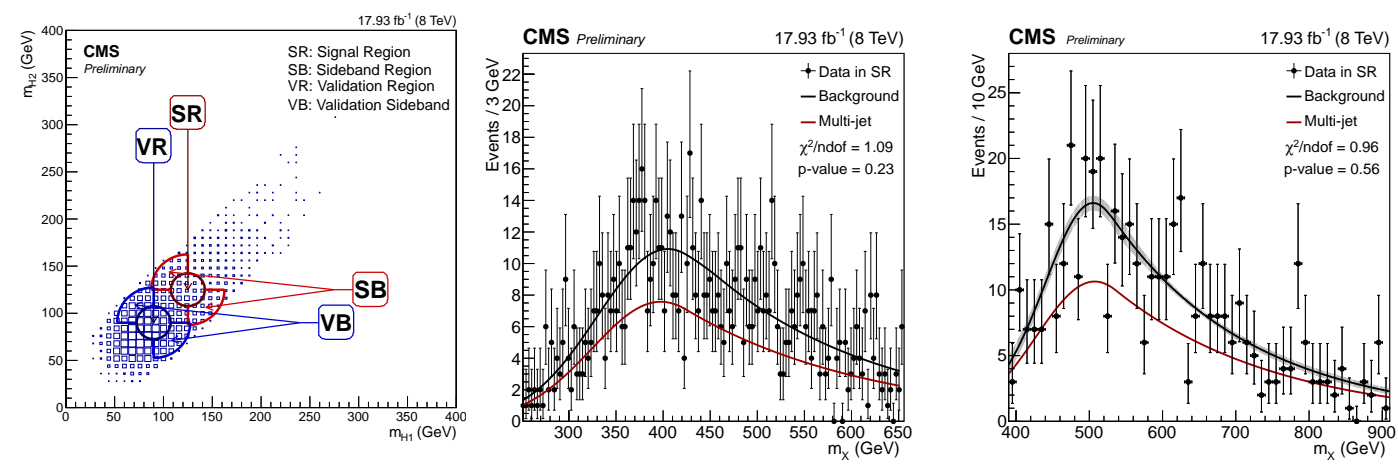

Figure 7: Illustration of the Signal (SR), Sidebands (SB) and Validation Regions (VR and VB) of the $h h \rightarrow$ $b \bar{b} b \bar{b}$ analysis (left). The four-body invariant mass in the low (center) and high mass scenario (right) [21].

The results of the two $X \rightarrow h h \rightarrow \gamma \gamma b \bar{b}$ and $X \rightarrow h h \rightarrow b \bar{b} b \bar{b}$ are reported in Fig. 8 for both spin- 0 and spin- 2 resonances and compared to the cross section of the resonances multiplied by the branching fraction to a pair of Higgs bosons, fixing some model parameters. No significant excess of events above the SM expectation is observed.
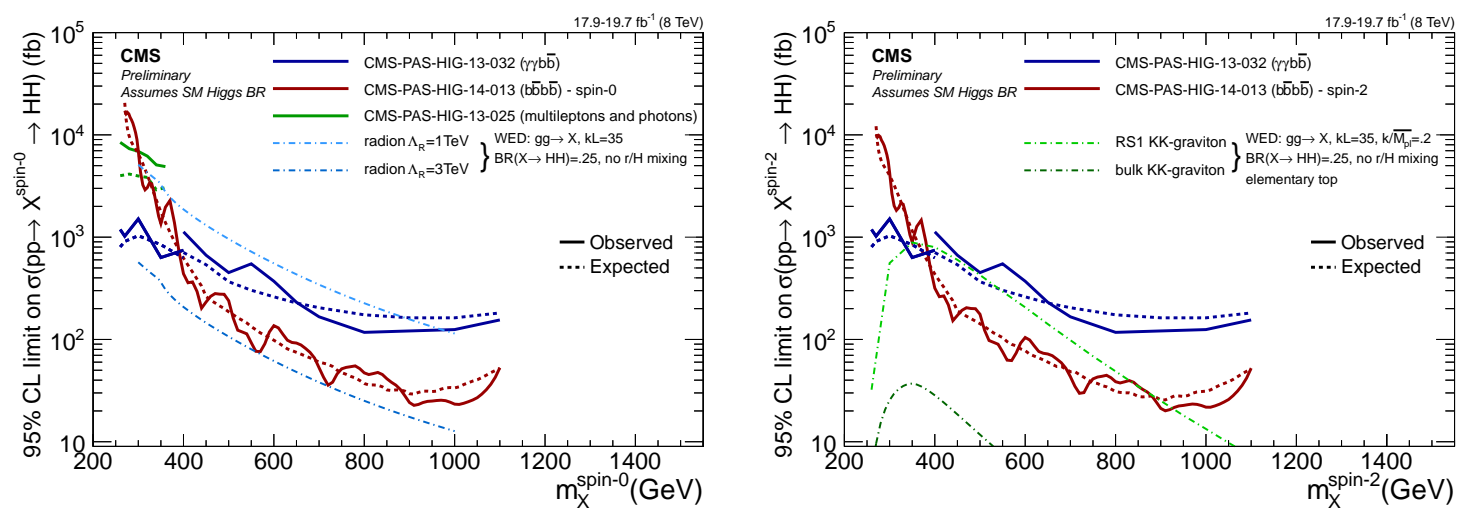

Figure 8: $95 \%$ CL expected and observed exclusion limit for the Radion (left) and Graviton (right). Blue lines show the results of the $\gamma \gamma b b$ analysis, the red ones the $b \bar{b} b \bar{b}$ and in green the sensitivity of the multilepton and diphoton analysis. Theory cross section predictions for certain model parameters are overlaid [21]. 


\section{Lepton Flavour Violating decays}

Even though Lepton-flavour violating (LFV) Higgs boson decays are not allowed in the SM, they can occur naturally in various BSM models such as composite-Higgs [11] and RandallSundrum models [8]. CMS looks for LFV decays $h \rightarrow \mu \tau$ of the $125 \mathrm{GeV}$ Higgs boson in the $\mu e$ and $\mu \tau_{h}$ final states, using $19.7 \mathrm{fb}^{-1}$ of $8 \mathrm{TeV}$ data [22]. Events are collected triggering and selecting isolated muons and electrons with opposite charge, and divided into jet-multiplicity categories to enhance the sensitivity to the different production modes. Since the muon is emitted from the Higgs and tends to have larger $p_{T}$ than in SM $h \rightarrow \tau \tau$ case, the Higgs boson candidate mass is reconstructed from the $\mu$, the visible $\tau$ products $\left(\tau_{v i s}\right)$ and the missing energy component along $\tau_{v i s}$ with an acceptable resolution, as shown in Fig. 9. The observed upper limit at 95\% CL on the $\mathrm{LFV} B(h \rightarrow \mu \tau)$ is $1.57 \%(0.75 \pm 0.38 \%$ expected $)$. Interpreted as signal, it corresponds to a $B(h \rightarrow \mu \tau)=0.89_{-0.37}^{+0.40} \%$ best fitted branching ratio, with a significance of $2.5 \sigma$. The result is reinterpreted as a constraint on the $\tau \mu$ Yukawa couplings, improving earlier indirect measurements by almost an order of magnitude.
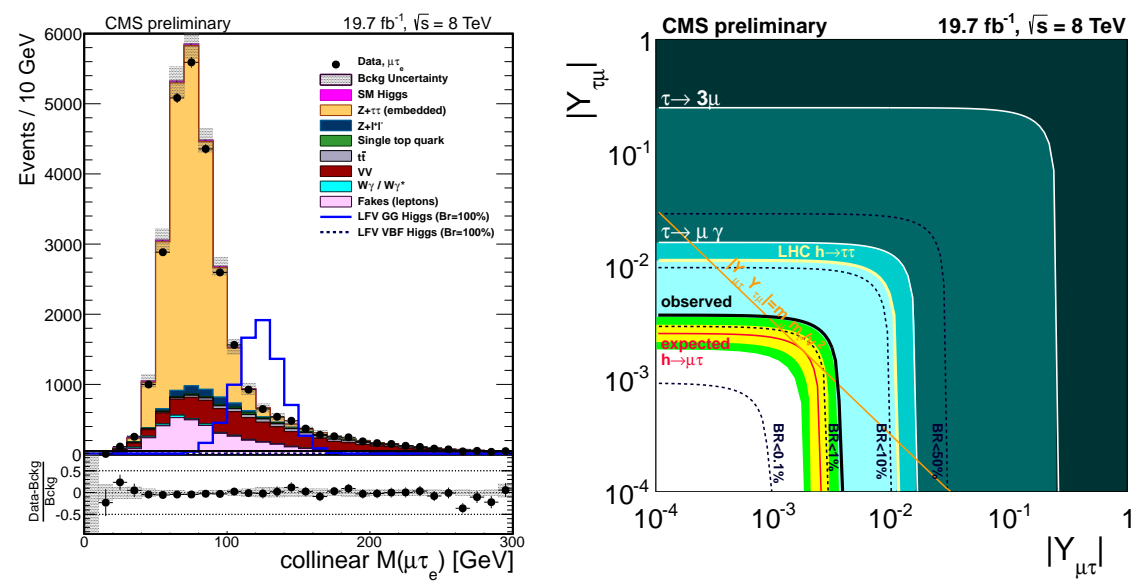

Figure 9: Left: collinear invariant mass in the $\mu+\tau_{e}$ final state. Right: constraints on the flavor violating Yukawa coupling $Y_{\mu \tau}$. The expected (red solid line) and observed (black solid line) limits are derived from the present analysis. The orange diagonal line is the theoretical naturalness limit $Y_{i j} Y_{j i} \leq m_{i} m_{j} / v^{2}$. Present constraint from direct searches, $\tau \rightarrow 3 \mu$ (dark green) and $\tau \rightarrow \mu \gamma$ (lighter green), are shown as well [22].

\section{Invisible Higgs boson decays}

A Higgs boson decaying with a significant probability into final states consisting entirely of invisible particles might be measurable if produced in association with other detectable particles. CMS searches this signature [23] for new physics in the Higgs boson associated production with a $Z$ boson, decaying into leptons or $b$-quarks, and in the Vector Boson Fusion (VBF), where two high energy forward jets, separated by a large rapidity gap, can be reconstructed. While the $Z \rightarrow$ $\ell \ell(\ell=e, \mu)$ and VBF searches rely on categorization and cut-based analyses, the $Z \rightarrow b \bar{b}$ uses a multivariate discriminator and an energy regression to the momenta of the two jets to further discriminate the backgrounds and increase the sensitivity. Invisible decays would manifest as an excess of events with high missing energy in the detector. No significant excess of events over the SM backgrounds is found, as shown if Fig. 10, and exclusion limits are interpreted in the Higgs 
portal model of Dark Matter (DM), assuming DM couples to the Higgs sector. Different scenarios are proposed, depending on the nature of the DM candidate.
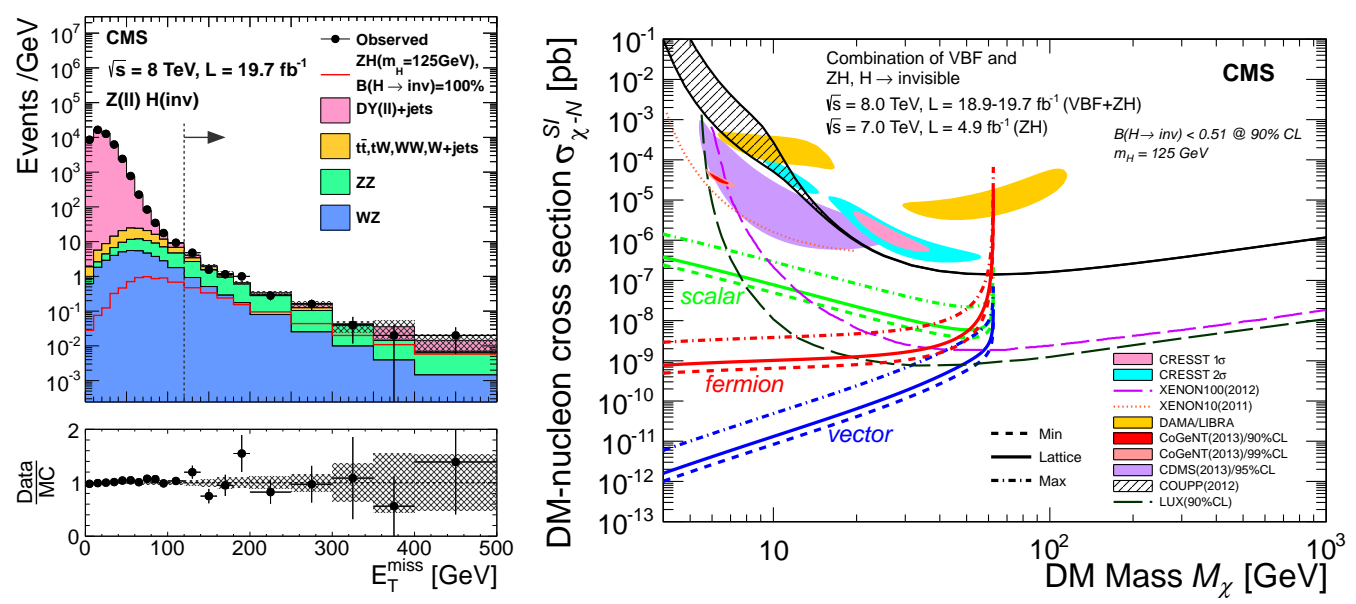

Figure 10: Left: distribution of the missing energy in data and background simulation in the $Z \rightarrow e, \mu$ channel. The expected signal corresponding to $\operatorname{Br}(H \rightarrow i n v)=100 \%$ is superimposed separately. Right: Upper limits on the spin-independent DM-nucleon cross section in Higgs-portal models, derived for $m_{H}=$ $125 \mathrm{GeV}$ and $\mathrm{Br}(H \rightarrow$ inv $)<0.51$ at $90 \% \mathrm{CL}$, as a function of the DM mass. Limits are shown separately for scalar, vector and fermion DM. Other experimental results are shown for comparison [23].

\section{Search for high mass bosons in the $\gamma \gamma$ channel}

The extension to higher masses of the searches of the SM Higgs boson is natural after the discovery of the $125 \mathrm{GeV}$ scalar. The $H \rightarrow \gamma \gamma$ channel [24] is one of the most promising channels, due to the excellent photon resolution of the CMS detector. Data, corresponding to the full 2012 luminosity, is collected with a double photon trigger. Events with at least two photon candidates are divided in categories depending on the geometrical regions of the electromagnetic calorimeter. No peaks over the smoothly falling diphoton invariant mass spectrum are found, and 95\% CL exclusion limits are set (Fig. 11), also as a function of the natural width of the resonance (up to $10 \%$ its mass) for the spin-0 and spin-2 hypothesis.

\section{Search for high mass bosons in the semileptonc WW channel}

A search for an heavy Higgs boson is performed in the $H \rightarrow W W$ channel [25]. While both $W$ bosons are on-shell, one leptonic decay $W \rightarrow \ell v(\ell=e, \mu)$ provides an easily triggerable final state, while the other $W \rightarrow q \bar{q}$ is an additional handle to discriminate against the dominating $W+$ jets background. The momentum of the neutrino is recovered from the missing energy and a kinematic fit requesting the $W$ invariant mass. A likelihood angular discriminator is built to enhance the signal significance. The four-body invariant mass is the variable to be fitted looking for the signal. No significant excess is found, and exclusion limits are interpreted in the Electronweak Singlet theory [10], where an additional scalar is introduced to the SM Lagrangian, and a new heavy scalar state $H$ is present, and mixes with the light $h$. The couplings of the SM particles with $H$ are different with respect to the SM case, and are parametrized by a factor $C^{\prime}$. The $C^{\prime}=1$ case is the SM-like 

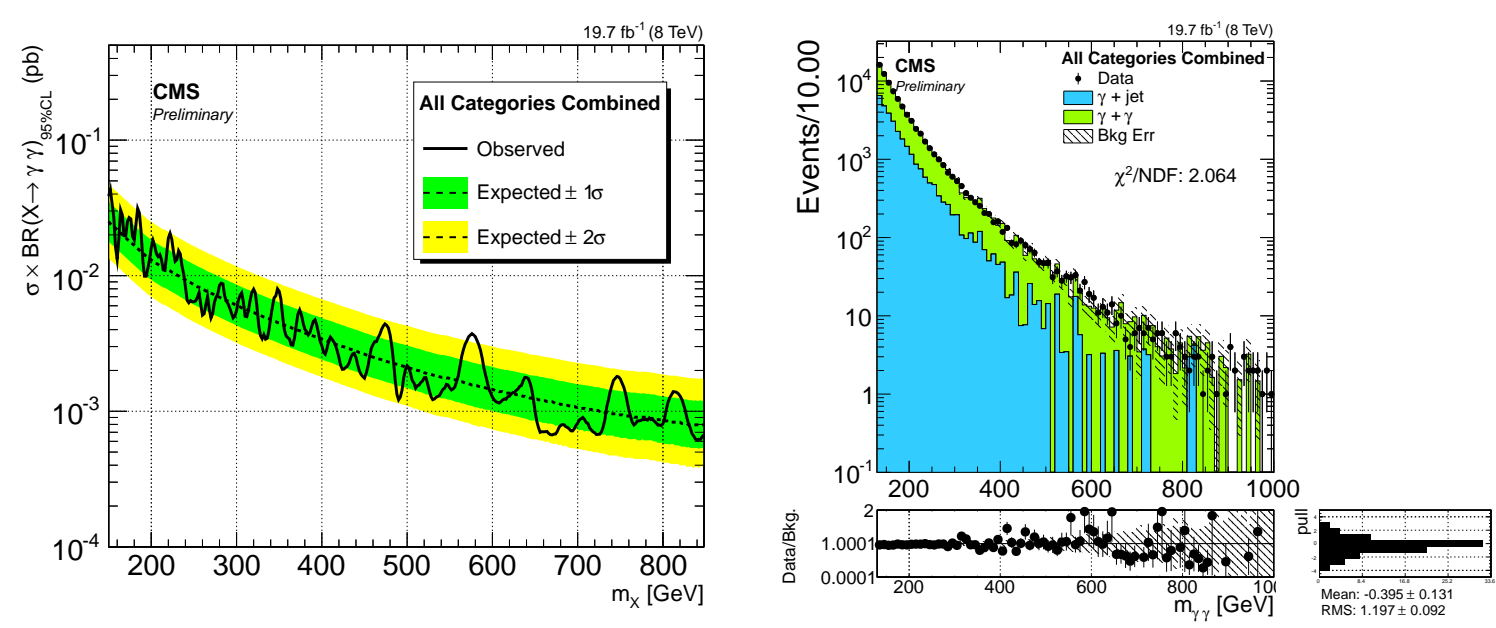

Figure 11: Left: Exclusion limit at 95\% CL on the cross section times the branching ratio of a spin 2 gluon fusion produced resonance decaying into two photons. Right: diphoton invariant mass for data and background simulation after selections [24].

scenario. An additional free parameter, $B r_{\text {new }}$, is introduced to take into account for $H$ decays into particles not predicted by the SM. The exclusion limits are reported in the $C^{\prime}-B r_{n e w}$ plane for different $m_{H}$ values, as shown in Fig. 12.
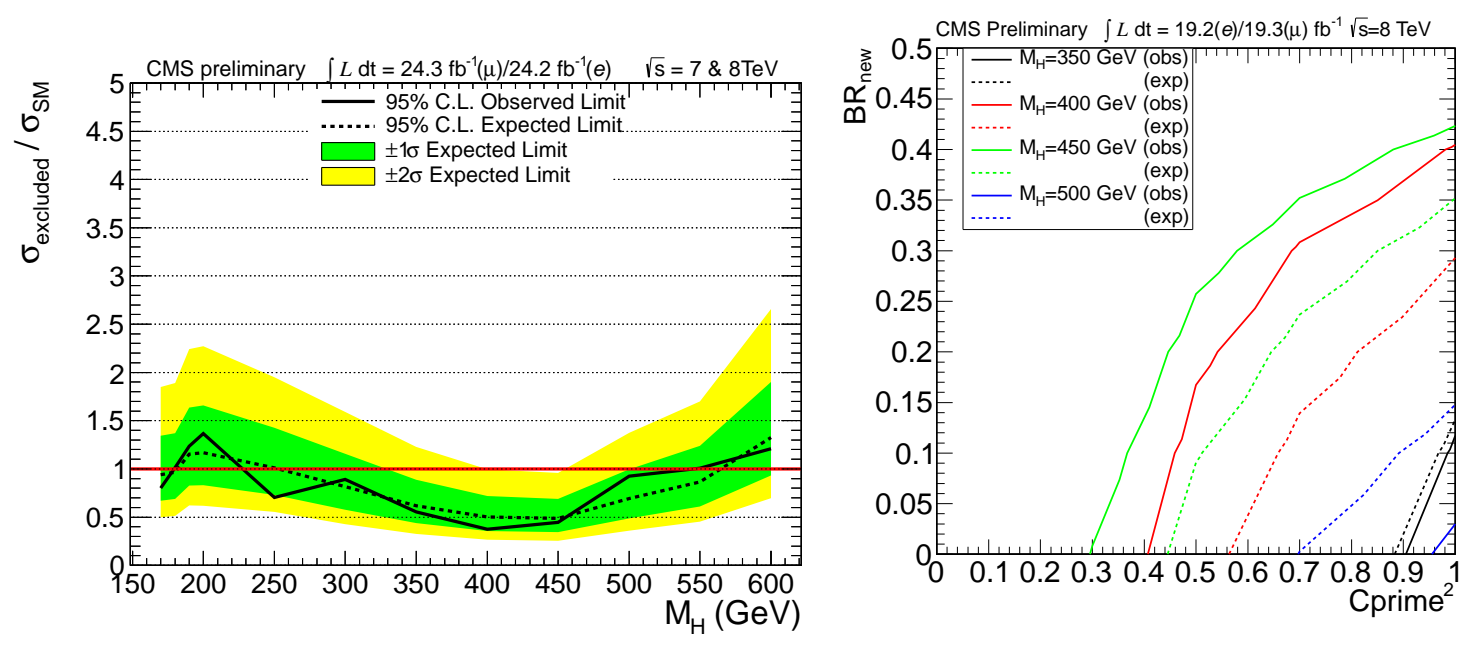

Figure 12: Left: observed and expected upper limit on the ratio of the production cross section to the SM expectation for the Higgs boson. The solid line at 1 indicates the SM expectation. The combined limit using both $7 \mathrm{TeV}$ and $8 \mathrm{TeV}$ data is shown. Right: $95 \%$ confidence exclusion curves taken from a grid scan in the $C^{\prime}$ vs. $B R_{\text {new }}$ space. Observed curves are drawn with solid lines and expected curves are drawn with dashed lines. The excluded region lies to the right and below the curves [25]. 


\section{Summary}

The $25 \mathrm{fb}^{-1}$ of data collected by the CMS experiment at $\sqrt{s}=7$ and $8 \mathrm{TeV}$, other than the fundamental discovery of a Higgs boson, provided several chances to investigate a plethora of possible new physics signatures. In this review, searches for an extended Higgs sector, exotic particles decaying in final states involving the SM Higgs boson, and non-SM Higgs boson decays have been discussed. No striking signatures of new phenomena have been observed, and stringent constraints on BSM theories parameter space have been set.

\section{References}

[1] The CMS Collaboration, Phys. Lett. B716 (2012) 30.

[2] The ATLAS Collaboration, Phys. Lett. B716 (2012) 1.

[3] The CMS Collaboration, JINST S08004 3-08, (2008)

[4] G. C. Branco et al., Phys. Rep. 516 1-2 (2012)

[5] S. P. Martin, Adv.Ser.Direct.High Energy Phys. 21 1-153 (2010)

[6] M. Carena et al., Eur. Phys. J. C 26, 601-607 (2003)

[7] U. Ellwanger et al., Phys. Rep., 496 1-2, (2010)

[8] L. Randall and R. Sundrum, Phys. Rev. Lett. 83 3370-3373 (1999)

[9] T. Gherghetta and A. Pomarol, Nucl.Phys. B586 141-162 (2000)

[10] R. Shrock, Phys. Rev. D 787 (2008)

[11] D. B. Kaplan, Nucl. Phys. B 254, 299 (1985)

[12] The CMS Collaboration, JHEP 10160 (2014)

[13] The CMS Collaboration, CMS-PAS-HIG-12-026, http://cds.cern.ch/record/1460104

[14] The CMS Collaboration, CMS-PAS-HIG-12-027, http://cds.cern.ch/record/1460105

[15] The CMS Collaboration, Phys. Lett. B 722, 4-5 (2013)

[16] The CDF Collaboration, Phys. Rev. D 85, 032005 (2012)

[17] The CMS Collaboration, CMS-PAS-HIG-12-011, http://cds.cern.ch/record/1453716

[18] The CMS Collaboration, CMS-PAS-HIG-13-010, http://cds.cern.ch/record/1563546

[19] The CMS Collaboration, CERN-PH-EP-2014-239 (2014)

[20] The CMS Collaboration, CMS-PAS-HIG-13-032, http://cds.cern.ch/record/1697512

[21] The CMS Collaboration, CMS-PAS-HIG-14-013, http://cds.cern.ch/record/1748425

[22] The CMS Collaboration, CMS-PAS-HIG-14-005, http://cds.cern.ch/record/1740976

[23] The CMS Collaboration, Eur. Phys. J. C 74 (8) 2980 (2014)

[24] The CMS Collaboration, CMS-PAS-HIG-14-006, http://cds.cern.ch/record/1714076

[25] The CMS Collaboration, CMS-PAS-HIG-13-027, http://cds.cern.ch/record/1743804 